\title{
The Influence of the Bitrate Level on the Subjective Sound Quality Perception of the Concatenated Non-Entropic Audio Coding Algorithms in the Digital Broadcasting Chain
}

\author{
Karel ZYKA \\ Czech Radio, Vinohradská 12, 12099 Praha 2, Czech Republic \\ karel.zyka@rozhlas.cz \\ Submitted May 14, 2020 / Accepted October 1, 2020
}

\begin{abstract}
Digital Audio Broadcasting (DAB) and all similar systems for digital radio and television broadcasting are inevitably associated with lossy psychoacoustic audio compression. The coding algorithms are continuously being improved. To achieve high sound quality a lower bitrate, required by the broadcasters, is now sufficient. This paper compares the relevant digital audio codecs: $M P E G 2$ and $A A C$ in three profiles (AAC LC, HE-AAC v1 and $H E-A A C$ v2). The well-known MP3 format is also added for a better comparison. A brief description of the basic functional principles of the codecs is followed by a comparison of their efficiency keeping in mind the subjectively comparable sound quality. The main contribution of this paper is the verification of the relationship of the bitrate level and sound quality in broadcasting environment and the finding out the influence of other, often more significant factors, such as the primary quality of the input recordings and the concatenation of non-entropic coding, on the subjective perception in the digital broadcasting chain. These findings are supported by the results of a unique research analysis providing an insight into which specific audio encoding configurations are used for DAB+ radio broadcasting in practice, in Europe as a whole and in individual European countries.
\end{abstract}

\section{Keywords}

DAB+, Digital Audio Broadcasting, psychoacoustic compression, coding, MPEG, MP3, AAC, HE-AAC, Spectral Band Replication, Parametric Stereo

\section{Introduction}

The use of a new technical standard for radio broadcasting, Digital Audio Broadcasting (DAB+) [1-4], is currently expanding intensively in Europe. DAB system is a linear digital distribution platform for the distribution of audio accompanied by additional multimedia content. It was officially established in February 1995, when it was standardized by the European Telecommunications Standards Institute (ETSI) as ETSI EN 300401 [2]. In February 2007, an improved version called DAB + [5] was released. Its main and crucial contribution was the application of a new, more efficient algorithm for encoding audio channels. $\mathrm{DAB}+$ system has brought a number of innovations and benefits. Compared to analogue FM broadcasting, it offers higher sound quality to listeners, better performance in complicated reception conditions, complementary data service and a wider range of radio stations.

These results were achieved mainly due to the following three state-of-the-art innovations:

1. Multiplexing, which associates individual radio programs into so-called multiplexes and then (in the case of $\mathrm{DAB}+$ ) encodes the transmission channel using concatenated encoding by Reed-Solomon code and by convolutional code - Forward Error Correction (FEC) [2]. FEC of each audio channel can be configured independently.

2. Coded Orthogonal Frequency Division Multiplexing (COFDM) modulation [6], which enables us to distribute the bitrate among a large number of parallel subcarriers. The symbol rate on each of them can then be relatively low. It allows the use of synchronous, single frequency networks (SFN) in wide geographical areas.

3. Psychoacoustic lossy digital audio coding [7] which uses the Advanced Audio Coding (AAC) algorithm with predictive analysis and with two superstructures: Spectral Band Replication (SBR) and Parametric Stereo (PS).

$\mathrm{DAB}+$ and generally all similar systems for digital broadcasting (DAB, DAB+, DRM, DRM+, HD Radio, CDR, DVB-T, DVB-T2 [8], DVB-S / DVB-S2) are based on the use of lossy, non-entropic audio codecs. The algorithms of these codecs work with relatively low bitrates. Moreover, these bitrates constantly decrease with codecs 
development (see Fig. 1). Non-entropic audio codecs perform data compression that exploits the psychoacoustic model of human hearing [9] and removes redundant data from the transmitted signal. These are the data that the human ear is unable to process usefully and thus they do not contribute to improving the sound quality. The signal processed in this way can no longer be fully restored to its original state (losslessly decoded), as with lossless formats [10], [11]: FLAC, ALAC, WavPack, Monkey's audio, etc., or with general compression methods: ZIP, RAR, etc. In terms of subjective perception of sound quality, which is decisive in the assessment of sound systems [12], it is possible to achieve (using the correct configuration of nonentropic audio codecs) such sound quality, which despite massive data reduction, can be subjectively perceived as virtually identical to the original [13-15]. It is therefore clear that for codecs used in the $\mathrm{DAB} / \mathrm{DAB}+$ system, there is no direct correlation between the bitrate and the sound quality. It always depends on the use of a particular coding algorithm.

In this paper, a complete overview of the $\mathrm{DAB}+$ audio coding is provided. The main contribution of this paper is:

- The verification of the relationship of subjective perception of the sound quality and the bitrate level in the digital broadcasting chain, using concatenated audio coding algorithms. This was done by conducting original blind listening tests described in Sec. 3.

- The unique research analysis of the DAB+ codec configurations used in practice in individual European countries, the results of which are in conformity with the findings in Sec. 3.

The paper is organized in the following manner: Section 2 presents the description of the audio codecs used in $\mathrm{DAB} / \mathrm{DAB}+$. Section 3 provides the comparison of their efficiency and discusses the influences on the perception of sound quality in the environment of the digital broadcasting chain. Section 4 analyzes and discusses the practical application of $\mathrm{DAB}+$ codec configurations in Europe and, finally, Section 5 concludes the paper.

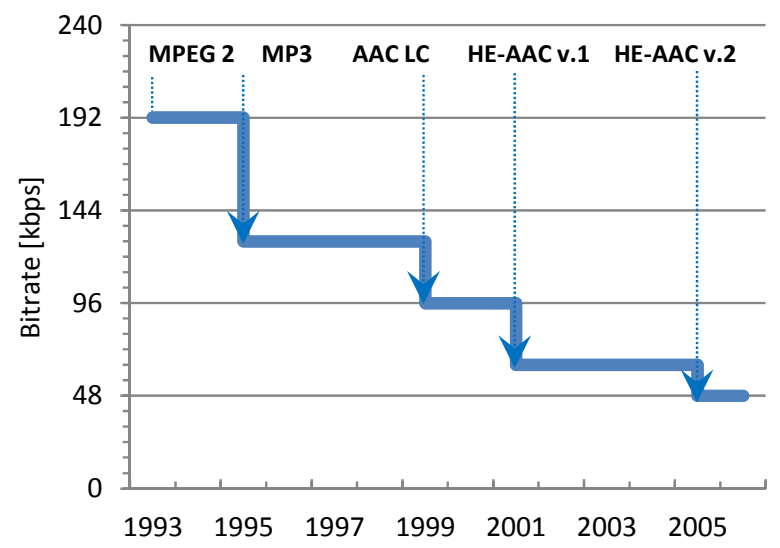

Fig. 1. Evolution of coding efficiency - the minimal bitrate to achieve the sound quality subjectively perceived as similar to the original signal.

\section{Description of the Audio Codecs Used in DAB / DAB+}

In the original DAB system the Moving Picture Experts Group (MPEG 2) codec [16] is applied. In the upgraded version $\mathrm{DAB}+$ the Advanced Audio Coding (AAC) codec [7] is used. AAC is available in three profiles: AAC LC, HE-AAC v1, HE-AAC v2. For comparison, the wellknown MP3 format [17] is included in the following overview.

\subsection{MPEG 2}

The oldest of these audio codecs is MPEG-1 Audio Layer 2 [16], [18], also known as MPEG 2 or MP2. It is part of the MPEG-1 Audio standard, including three encoding algorithms known as Layer 1 (MP1), Layer 2 (MP2) and Layer 3 (MP3), all of which were standardized at the same time in 1993 as ISO/IEC 11172-3 [19]. MPEG 2 (like all three codecs of this standard) is based on the previous Masking pattern adapted Universal Subband Integrated Coding and Multiplexing (MUSICAM) codec [20] developed by the German "Institut für Rundfunktechnik" (IRT) in 1989.

MPEG 2 is a subband audio encoder in which the bitrate is reduced by psychoacoustic coding in the time domain using a filter bank. At the input of the encoding process, the original signal is divided into 32 subbands using band-pass filters. A psychoacoustic model [9] is then applied to such a modified signal. In the subsequent quantization, it takes into account the characteristics of human hearing when perceiving near-frequency components. The process called frequency masking [9] takes advantage of the fact that if a strong signal of a certain frequency sounds simultaneously with a weaker signal at a frequency close to the stronger one, the human hearing cannot perceive the weaker signal unless it exceeds the threshold i.e. the intensity level set by the masking curve. The encoder then ignores such components that do not reach this threshold, and can use the data capacity to process the useful, audible part of the signal. Thus, if the sound in any of the 32 subbands is evaluated by the psychoacoustic model to be imperceptible, the entire subband is excluded from encoding in that period. Removing the masked components practically does not affect the resulting sound perception.

MPEG 2 has brought a significant progress in its time by enabling digital audio to be compressed in a wide range of options from $8 \mathrm{kbps}$ to $384 \mathrm{kbps}$ at sampling rates from $16 \mathrm{kHz}$ to $48 \mathrm{kHz}$. It also offers four operating modes: mono, stereo, dual channel and joint stereo. Using a bitrate range from $192 \mathrm{kbps}$ to $256 \mathrm{kbps}$, a high quality sound can be achieved for a stereo signal, which is subjectively perceived as comparable to the so-called "CD quality". Studio quality of a stereo signal corresponds to a bitrate of 384 kbps. Until recently, MPEG 2 has been used (and in some cases is still in use) for digital radio and television broadcasting. It is a part of the original version of $\mathrm{DAB}$, 
but also DRM, DVB-T and DVB-S. MPEG 2 is also one of the audio formats used by DVD.

\subsection{MP3}

MP3, or MPEG-1 Audio Layer 3 [21], [17], is another audio codec of this standard. It was also introduced in 1993, but due to the high demands on hardware, its use began to expand gradually only from 1995. The core of its algorithm, as well as the psychoacoustic characteristics and the format structure, are based on the MPEG 2 codec. Unlike the MPEG 2 codec, MP3 is a transform codec operating in the frequency domain to which, after conversion from the time domain, it transforms the input signal in the form of 576 frequency components. This represents a significantly finer frequency resolution and thus the psychoacoustic model can be applied more selectively and more effectively. The higher efficiency of the MP3 algorithm is also due to the fact that the algorithm uses temporal masking [9] in addition to frequency masking. In temporal masking, the redundant signal components of closely consecutive sounds are eliminated. A strong signal masks the weak signal that immediately follows it (post-masking), but also the weak signal immediately preceding it (pre-masking). Both of these components, if they have parameters determined by the psychoacoustic model, cannot be recognized by human hearing and can therefore be eliminated without affecting the resulting perception of sound.

While MPEG 2 has become the standard for radio and television broadcasting, MP3 has become extremely popular in the areas of computers, media players and Internet applications, and has significantly influenced the evolution of the phonographic industry.

\subsection{AAC}

Advanced Audio Coding (AAC) [7], [22], [23] represents the state-of-the-art and currently also the most efficient audio codec for lossy digital compression. A number of companies participated in its development: AT\&T Bell Laboratories, Fraunhofer Institute, Dolby Laboratories, Sony, Nokia, LG Electronics, NEC, NTT Docomo, ETRI, JVC Kenwood, Philips, Microsoft and Panasonic. The basic version of the AAC was first introduced in 1997 as a part of the MPEG-2 Part 7 standard (ISO / IEC 13818-7) [24]. In 1999, the whole family of improved AAC profiles became part of the MPEG-4 Part 3 standard (ISO/IEC 14496-3) [25]. AAC is implemented in the upgraded version of $\mathrm{DAB}(\mathrm{DAB}+)$, but also in other digital radio and television platforms (DRM+, DVB-T2, DVB-S2) and in a variety of audio and audiovisual devices (iPhone, iPod, iPad, iTunes, Nintendo, PlayStation, etc.).

The base of the AAC audio codec family is the Low Complexity (LC) profile. It can be complemented by two superstructures: Spectral Band Replication (SBR) and Parametric Stereo (PS) that make its coding even more efficient. Depending on the combination of their use, they are referred to as HE-AAC v1 (AAC LC + SBR) or HE-AAC v2 (AAC LC + SBR + PS). The total bitrate of the HE-AAC codec is thus the sum of the bitrates of the core codec and of one or both superstructures, where both superstructures use a relatively small part of the data capacity. The resulting coding efficiency of HE-AAC v2 is then at least four times higher than that of the older codec MPEG 2. Using this combination, the subjectively perceived high sound quality can be achieved at $48 \mathrm{kbps}$.

\subsubsection{AAC LC}

Like its predecessor MP3, AAC is a transform codec operating in the frequency domain. AAC also uses the same encoding principles like MP3, but offers many improvements using a number of new additional encoding tools.

The basic profile of the AAC family (core codec) is referred to as Low Complexity (AAC LC) [7], [23]. The audio signal is converted from the time domain to the frequency domain using the Modified Discrete Cosine Transform (MDCT) method [26]. This is followed by quantization, which is based on a psychoacoustic model (similar to MPEG 2 and MP3) and then by data insertion for error correction. However, compared to MP3, the algorithm of $\mathrm{AAC}$ has a higher frequency resolution, improved joint stereo coding and improved lossless Huffman coding. Instead of a hybrid (cascaded) filter bank, which is used in MP3, AAC uses a standard overlapped MDCT filter bank and can use Temporal Noise Shaping (TNS) [27] to control the temporal shape of the quantization noise in the time domain. The data for this shaping are derived by predictive frequency domain signal analysis.

With many minor enhancements to increase encoding efficiency, AAC achieves on average the same sound quality as MP3 at approximately $70 \%$ bitrate. The difference is particularly evident at the low bitrates for which the AAC is primarily designed.

\subsubsection{HE-AAC v1}

The first superstructure of the AAC codec is Spectral Band Replication (SBR) [23], [28], [29], developed by Coding Technologies AB in 2001. The profile combining AAC LC and SBR is then referred to as High Efficiency AAC Version 1 (HE-AAC v1) [22], [23]. When the SBR is active, only the lower part of the frequency spectrum, which provides more information for sound perception [23], [30], is coded and transmitted. The core codec works at half the original sampling rate (which usually means at $24 \mathrm{kHz}$ ). Higher frequencies (usually above $12 \mathrm{kHz}$ ) are then coded using the SBR superstructure and the full bandwidth is reconstructed in the receiver decoder.

When coding audio much of the bitrate is used for the processing of high frequencies. From the psychoacoustic point of view, however, these are of relatively low importance, especially the last two octaves of the sound spectrum. The principle of SBR is based on this fact, which 
is further based on the knowledge that there is a strong correlation between the lower and upper part of the audio spectrum (referred to as the "high band" and the "low band"). Thanks to this, the upper part of the frequency spectrum of the audio signal can be approximated by transposing its lower part and by recalculating the signal envelope. In SBR, the transposition is controlled by SBR data, such as the high band signal spectral envelope, that help reconstruct the spectral components in high band region.

During encoding, the input signal is analyzed and then the spectral envelope and the characteristics of the high band of the frequency spectrum in relation to the low band are calculated. The resulting SBR data are then multiplexed (together with the core stream data) into a single bitrate. The SBR data use a bitrate of only $1 \mathrm{kbps}-3 \mathrm{kbps}$ per audio channel.

During decoding, the common bitrate is first demultiplexed and then core decoding is performed. The SBR data are then applied to the resulting signal, controlling the Spectral Band Replication process. The result of this process is a full bandwidth signal. This method ensures partial backward compatibility, so that if such signal is decoded on AAC devices that are not equipped with SBR functionality, the signal will still be decodable. However, compared to a full SBR decoder, its frequency range will be limited to the low band of the spectrum corresponding to half the sampling frequency.

\subsubsection{HE-AAC v2}

This profile introduces the second superstructure of the AAC codec, Parametric Stereo (PS) [23], [30], [31], which dates from 2005. The mode, referred to as High Efficiency AAC Version 2 (HE-AAC v2), uses both superstructures SBR and PS. In this case, only monophonic sum signal of the left and right channel is encoded and additional stereo descriptive PS data are transmitted through the channel. The resulting stereo image is then reconstructed in the receiver decoder.

When coding a stereo signal, the stereo image is analyzed first and then converted to a parametric representation, which describes the time and phase differences and correlations between stereo channels. As a result, it is not necessary to encode and transmit both channels, but only their monaural representative, which is supplemented by the parameters necessary to reconstruct the original stereo image. The parametric PS data use a bitrate of only 2 kbps-3 kbps.

The advantage of the highest AAC profile is its modularity. The individual superstructures can be selectively deactivated, depending on the desired application and conditions, and the codec can be changed up to a basic fullbandwidth AAC LC profile with the independent channels. HE-AAC v2 is partially backward compatible with both HE-AAC v1 (at the cost of losing stereo information) and AAC LC (where bandwidth is limited in addition to the loss of the stereo image). However, the signal can be decoded in both cases.

\subsection{The Use of the Codecs in Contemporary Environment}

If we look at the codecs discussed from the perspective of their current use, we can say that:

- MPEG 2 performs the least invasive sound modification and at higher bitrates (above $256 \mathrm{kbps}$ ) provides high sound quality. It is advantageous where the bitrate is not the issue and where additional digital processing of audio and thus the concatenation of lossy codecs is expected. Typical example is rebroadcasting (broadcasting of content that will then be retransmitted).

- MP3 remains the dominant format for storing and sharing music by end users. It generally replaced previous personal music storage systems.

- AAC represents a modern alternative to MP3 for music use by the end users and is also a standard in contemporary digital broadcasting systems such as $\mathrm{DAB}+, \mathrm{DRM}+$ and DVB-T2. Its innovated version, High Efficiency AAC, is the state-of-the-art technology that offers high sound quality even at very low bitrates.

\section{Comparison of the Efficiency of DAB / DAB + Audio Codecs and their Influence on the Perception of Sound Quality}

As can be seen from the descriptions of the individual audio codecs, they differ not only by the used coding algorithms, but also by their efficiency, i.e. by the degree of the bitrate reduction while maintaining similar subjectively perceived sound quality. Therefore, the bitrates used for audio coding can never be judged alone (in relation to the sound quality), but always only in connection with the particular type of audio codec used. Figure 2 shows a comparison of the bitrates necessary for the individual audio codecs to achieve the kind of sound quality that is subjectively perceived as similar to the original, uncompressed signal. While MP2 requires $192 \mathrm{kbps}-256 \mathrm{kbps}$ to achieve this, $128 \mathrm{kbps}-192 \mathrm{kbps}$ is enough for MP3, and the AAC LC codec only needs $96 \mathrm{kbps}-128 \mathrm{kbps}$. If the SBR superstructure of HE-AAC v1 is active, $64 \mathrm{kbps}-96 \mathrm{kbps}$ is sufficient to achieve the same subjective results, if both SBR and PS superstructures of HE-AAC v2 are active, the required bitrate reduces to $48 \mathrm{kbps}-64 \mathrm{kbps}$.

These assumptions were verified before the start of the regular DAB+ broadcasting of Czech Radio using the MUSHRA blind listening tests, in accordance with ITU-R Recommendation BS.1534-3 [12]. The tests [32] were conducted in May 2015 in the recording studio R1 (equipped with Studer Vista 9 mixing console, Digital Audio Workstation ProTools 10 and Genelec 1038 audio monitors). The listening panel [33] was composed of twenty 


\begin{tabular}{|l|l|l|l|l|l|}
\hline \multicolumn{1}{|c|}{ Bitrate [kbps] } & $48-$ & $64-$ & $96-$ & $128-$ & $192-$ \\
Audio Codec & -64 & -96 & -128 & -192 & -256 \\
\hline MPEG 2 & & & & & \\
\hline MP3 & & & & & \\
\hline AAC LC & & & & & DAB \\
\hline HE-AAC v1 & & & & & \\
\hline HE-AAC v2 & & & & & \\
\hline
\end{tabular}

Fig. 2. Bitrates necessary for the individual audio codecs to achieve the sound quality subjectively perceived as similar to the original signal.

experienced assessors with sufficient experience in critical sound assessment and the ability of minor impairment recognition. They were aged 21 to 59 years with an average of 37 years. Before the blind listening test, the assessors were trained on the procedure and the content of the test itself. The training also included demonstrations of the kinds of sound impairments to be detected when listening, i.e. impairments caused by lossy audio coding, compared to the original reference signal. The critical material tested represented a typical radio program and consisted of a total of ten music samples of different genres $(1 \times$ rock, $3 \times$ pop, $3 \times$ classic, $1 \times$ electronic, $1 \times$ jazz and $1 \times$ spoken word $)$ and of different character in terms of signal dynamics and spectral density. The length of the excerpts was 10-15 seconds. Each sample was processed with seven different modifications: original reference from the radio archive (WAV $24 \mathrm{bit} / 48 \mathrm{kHz}$ ), AAC LC with a bitrate of $128 \mathrm{kbps}$, HE-AAC v1 with a bitrate of $96 \mathrm{kbps}$ and $80 \mathrm{kbps}, \mathrm{HE}-\mathrm{AAC}$ v2 with a bitrate of $64 \mathrm{kbps}$ and $48 \mathrm{kbps}$ and MPEG 2 with a bitrate of $256 \mathrm{kbps}$. Two anchors were used. A hidden reference in the form of an unprocessed signal and a low anchor in the form of the $3.5 \mathrm{kHz}$ low pass filtered original signal. All presented material was stereo.

The test confirmed that when the recommended bitrates are used for the relevant audio codecs, high subjectively perceived sound quality is achieved in all instances. The assessors chose the samples coded with HE-AAC v1 at a bitrate of $80 \mathrm{kbps}$ as the samples of the best subjective sound quality. The second subjectively best evaluated samples were coded with HE-AAC v2 at a bitrate of $48 \mathrm{kbps}$.

The tests have also shown that subjective perception of sound quality is more influenced by the configuration and parameters of the entire digital broadcasting chain than by the bitrate of the codec used. The digital broadcasting chain consists of a series of separate operations (phases) that intensively manipulate an audio signal. The first phase is usually represented by distribution media of music records (CD, MP3, etc.). The second phase is signal processing in studio computer systems. The third phase is the processing of the audio signal by the output broadcast audio processor [34], [35] and the fourth phase is the digital transmission itself [13], [32]. In each of these phases, non-entropic data reduction (lossy coding) usually is or can be used. Their multiple cascading and transcoding is then unavoidable. Moreover, this concatenation usually uses different coding algorithms and their parameters. The result is a multiple exposure of lossy coding to the audio signal, the impact of which affects the subjectively perceived sound quality.

Even though the topic of DAB and related audio codecs is already well researched, previous works have not yet examined them from the perspective of the entire broadcast system as a comprehensive specific environment. This research shows that the perceived sound quality within the entire transmission chain is influenced mainly by three key factors:

- The first factor is the technical quality of the input signal (e.g. music records).

- The second factor represents the changes of the audio signal by the multiple cascading of the lossy non-entropic audio codecs with transcoding of the signal within the entire digital broadcasting chain.

- The third factor is the correct choice of codec configurations and their respective parameters.

\section{Analysis of the Audio Codec Configurations Used for DAB+ in Europe}

$\mathrm{DAB}+$ allows audio encoding using three AAC codec profiles, each with a wide range of applicable bitrates. The following analysis explores what configurations are actually used for $\mathrm{DAB}+$ audio coding in individual European countries and what configurations, in general, are typical for $\mathrm{DAB}+$ in practice. The analysis was carried out in February 2020 using the FMLIST database [36] and the DAB Ensembles Worldwide database [37], as well as the information from WorldDAB [38] and from the European Broadcasting Union (EBU) - Digital Radio Report 2020 [39]. Where necessary, the data were further refined using the information sources of national DAB+ operators. The analysis included ten European countries (Top 10) whose $\mathrm{DAB}+$ broadcasting can be considered as the most developed and established, both in terms of the DAB radio industry development (signal coverage, the number of radio stations operated and the listening audience), and in terms of the related legal environment [38]. To ensure the validity of the data and to take into account the difference between nationwide and regional stations, each presence of a radio station in each single frequency network was counted separately in the analysis.

\subsection{Summary Results}

The overall results of the analysis (see Fig. 3) show that, in the European context, the HE-AAC v1 codec with SBR superstructure is used most frequently, in $82 \%$ of cases. 


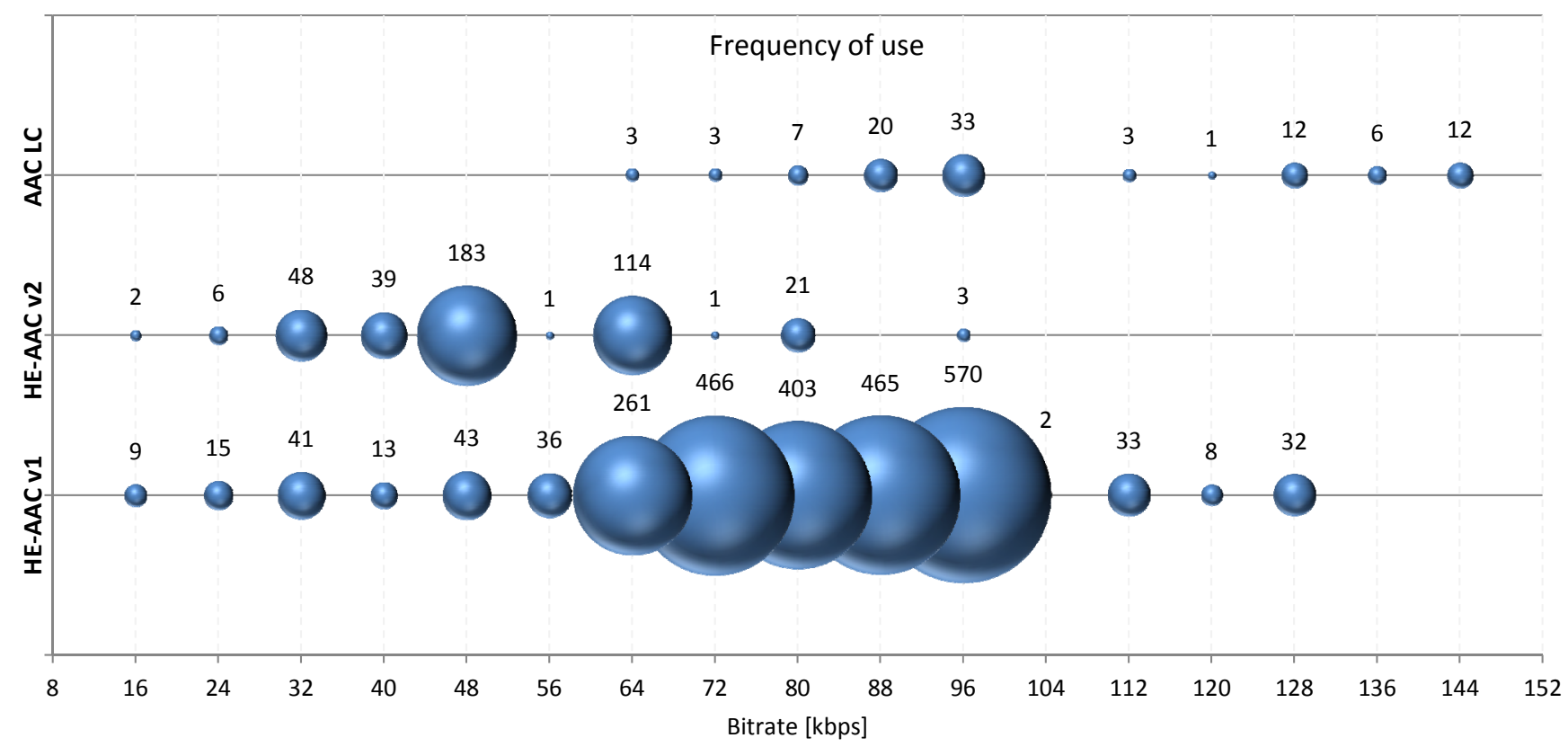

Fig. 3. Frequency of use of the individual bitrate levels for $\mathrm{DAB}+$ audio coding in Europe, displayed separately for each $\mathrm{AAC}$ profile.

The HE-AAC v2 codec with both superstructures (SBR and PS) also has a significant $15 \%$ share. The basic profile of AAC (AAC LC) is used in only $3 \%$ of the cases.

On closer inspection, it can be seen that $84 \%$ of all radio stations are coded with one of the seven most common configurations. The most frequently used, with a very balanced share, are four HE-AAC v1 codec configurations with $96 \mathrm{kbps}(20 \%), 72 \mathrm{kbps}(16 \%), 88 \mathrm{kbps}(16 \%)$ and $80 \mathrm{kbps}(14 \%)$ bitrates, followed by the same codec with a bitrate of $64 \mathrm{kbps}(9 \%)$, and two HE-AAC v2 codec configurations with $48 \mathrm{kbps}(6 \%)$ and $64 \mathrm{kbps}(4 \%)$ bitrates. As shown in Fig. 3, a further 28 audio codec configurations are used in practice for $\mathrm{DAB}+$, but the frequency of their use is marginal and rarely reaches $1 \%$.

These statistical results confirm that the state-of-theart audio coding (HE-AAC v1, HE-AAC v2) is used for $\mathrm{DAB}+$ dominantly, almost exclusively in practice. Despite low bitrates, it is used for broadcasting of all genres.

The results also show that the configurations of these codecs, across all European countries in the test, fall into the bitrate range where they provide maximum efficiency and sound quality, which is subjectively almost indistinguishable from the original sound. In the case of HE-AAC v1, it is a bitrate in the range of $64 \mathrm{kbps}-96 \mathrm{kbps}$, as discussed in Sec. 3. We can see that in practice there is neither oversizing with higher bitrates, which is inefficient, nor undersizing with lower bitrates, where the interfering artifacts can already be perceived.

The differences in the use of specific bitrates (in the range of optimal bitrates), which is visible in individual countries, are influenced by the fragmentation of the individual national markets. The choice of the specific bitrate is then influenced mainly by the capacity of individual $\mathrm{DAB}+$ networks and by the level of use of supplementary multimedia data. However, the different types of content broadcasted by radio stations in the $\mathrm{DAB}+$ in the individual countries also have an effect. The complexity of broadcast content is decisive (classical music at the upper end of the bitrate range and spoken word at the lower).

The graphs also show the differences between countries in terms of uniformity. This is due to the different structure of the network operators and due to the balance of the number of private and public broadcasters. While, for example, France or Belgium have dominant operators, in the Netherlands or Denmark their composition is much more varied.

\subsection{Detailed View of the Individual European Countries}

Looking at the individual countries of the researched European group (Top 10), it can be seen that the approach to $\mathrm{DAB}+$ audio coding is not uniform and that there are major differences. In principle, they can be divided into three groups. The first group includes the countries with one significantly dominant standard (France, Belgium). The second group includes the countries where strong preferences of several codec configurations are evident. These are mostly three to four variants (Norway, Switzerland, Germany, Great Britain, the Netherlands and Austria). The third group consists of countries where, despite the obvious preference of several dominant approaches, the audio coding is distributed among multiple configurations (Denmark, Italy).

The obvious dominant encoding in France is HE-AAC v1 with a bitrate of $88 \mathrm{kbps}$, which is used by $89 \%$ of radio stations. The remaining four configurations that occur there are marginal, each with less than 5\% share (see Fig. 4). 
A similar situation is in Belgium. The clearly dominant encoding there is HE-AAC v1 with a bitrate of $96 \mathrm{kbps}$, which is used by $88 \%$ of radio stations. The other four configurations are also marginal, again each with less than $5 \%$ share (see Fig. 5).

In Switzerland, $94 \%$ of radio stations are coded with one of the three most common configurations listed below. In all three cases the HE-AAC v1 codec is used. The most widely used bitrate is $72 \mathrm{kbps}(55 \%)$, followed by $64 \mathrm{kbps}$ (29\%) and $80 \mathrm{kbps}(11 \%)$. Seven other marginal configurations are in operation, but they always reach a maximum of $1 \%$ (see Fig. 6).

Similarly, $94 \%$ of radio stations in Austria are coded with one of the three most common configurations. The most used is the HE-AAC v1 codec with a bitrate of $72 \mathrm{kbps}(68 \%)$, followed by the HE-AAC v2 codec with $40 \mathrm{kbps}(18 \%)$ and the HE-AAC v1 codec with $80 \mathrm{kbps}$ $(8 \%)$. The remaining three configurations do not exceed $3 \%$ of each occurrence (see Fig. 7).

The preference of the three dominant configurations can also be observed in the Dutch DAB+ broadcasting. These cover $82 \%$ of all radio stations. The most commonly used codec is HE-AAC v1 with a bitrate of $64 \mathrm{kbps}(46 \%)$, followed by HE-AAC v2 with $48 \mathrm{kbps}$ (25\%) and HE-AAC v1 with $96 \mathrm{kbps}(11 \%)$. The remaining six configurations do not exceed $5 \%$ of each occurrence (see Fig. 8).

In Norway, $81 \%$ of $\mathrm{DAB}+$ radio stations are encoded in one of the following four configurations. The codec HE-AAC v1 with a bitrate of $80 \mathrm{kbps}(57 \%)$ is in the dominant position, followed by the same codec with $64 \mathrm{kbps}(9 \%)$ and $96 \mathrm{kbps}(9 \%)$. The fourth most common is the HE-AAC v2 codec with a bitrate of $48 \mathrm{kbps}(6 \%)$. There are 15 other configurations in operation, but they do

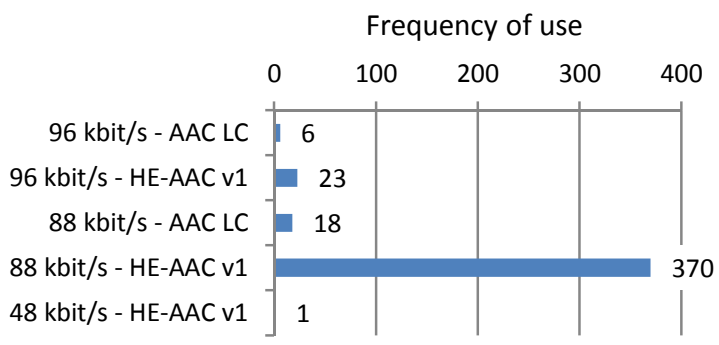

Fig. 4. Configurations used for $\mathrm{DAB}+$ audio coding in France and their respective frequency of use.

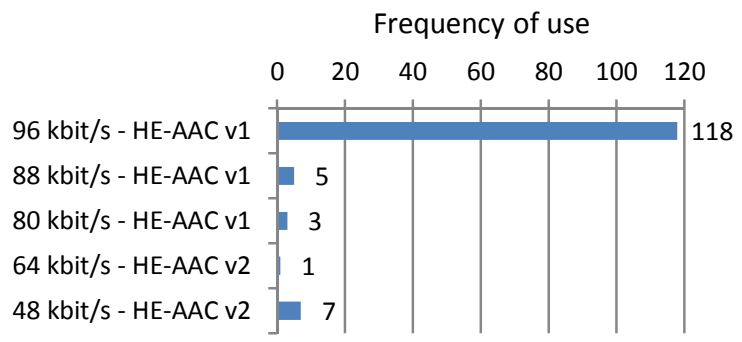

Fig. 5. Configurations used for $\mathrm{DAB}+$ audio coding in Belgium and their respective frequency of use.

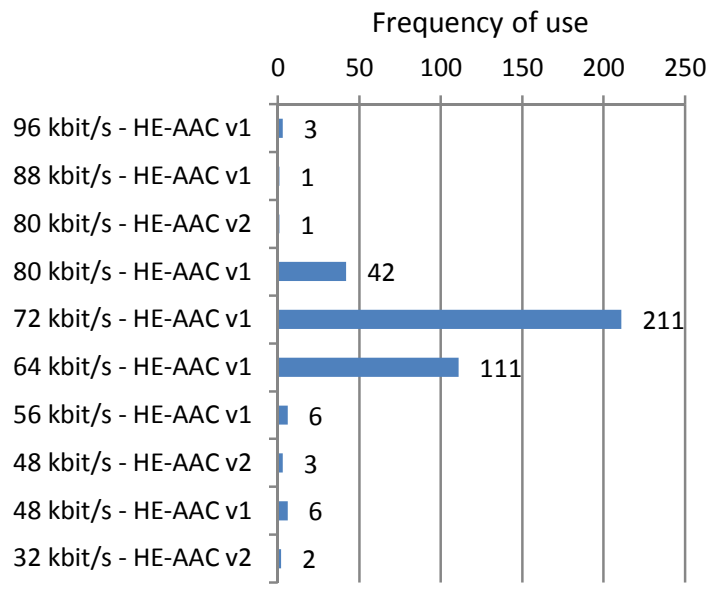

Fig. 6. Configurations used for $\mathrm{DAB}+$ audio coding in Switzerland and their respective frequency of use.

\section{Frequency of use}

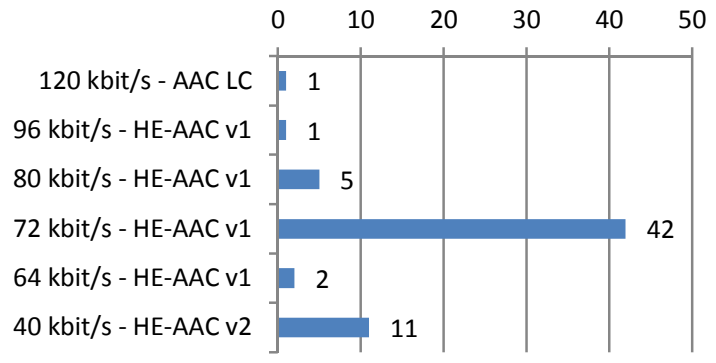

Fig. 7. Configurations used for $\mathrm{DAB}+$ audio coding in Austria and their respective frequency of use.

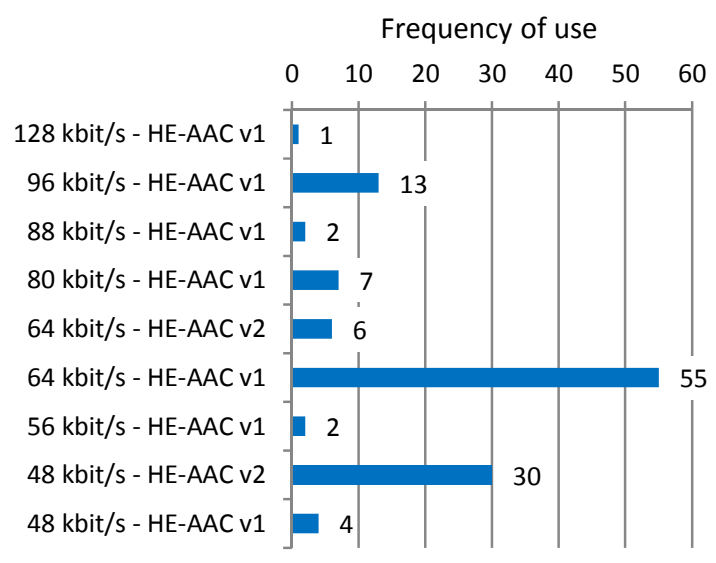

Fig. 8. Configurations used for $\mathrm{DAB}+$ audio coding in the Netherlands and their respective frequency of use.

not exceed 3\% of each occurrence (see Fig. 9). In addition to $\mathrm{DAB}+$, the original version of $\mathrm{DAB}$ with the MPEG 2 codec is still in operation in Norway. Its occurrence is, however, very marginal.

In Germany, $83 \%$ of radio stations are coded with one of the four most common configurations as well. In all four cases the HE-AAC v1 codec is used. The most widely used bitrate is $96 \mathrm{kbps}(35 \%)$, followed by $72 \mathrm{kbps}(26 \%)$ and $80 \mathrm{kbps}(14 \%)$. The fourth most commonly used is the $88 \mathrm{kbps}(9 \%)$ bitrate. There are 17 other configurations in operation, but they do not exceed $4 \%$ of each occurrence (see Fig. 10). 


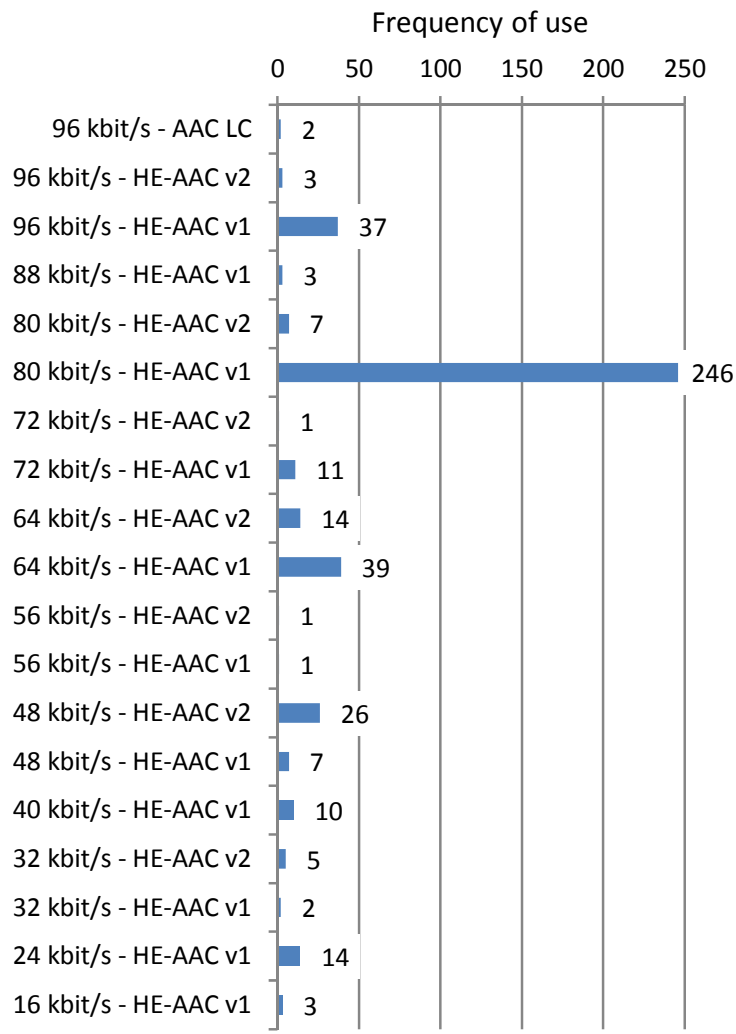

Fig. 9. Configurations used for $\mathrm{DAB}+$ audio coding in Norway and their respective frequency of use.

\section{Frequency of use}

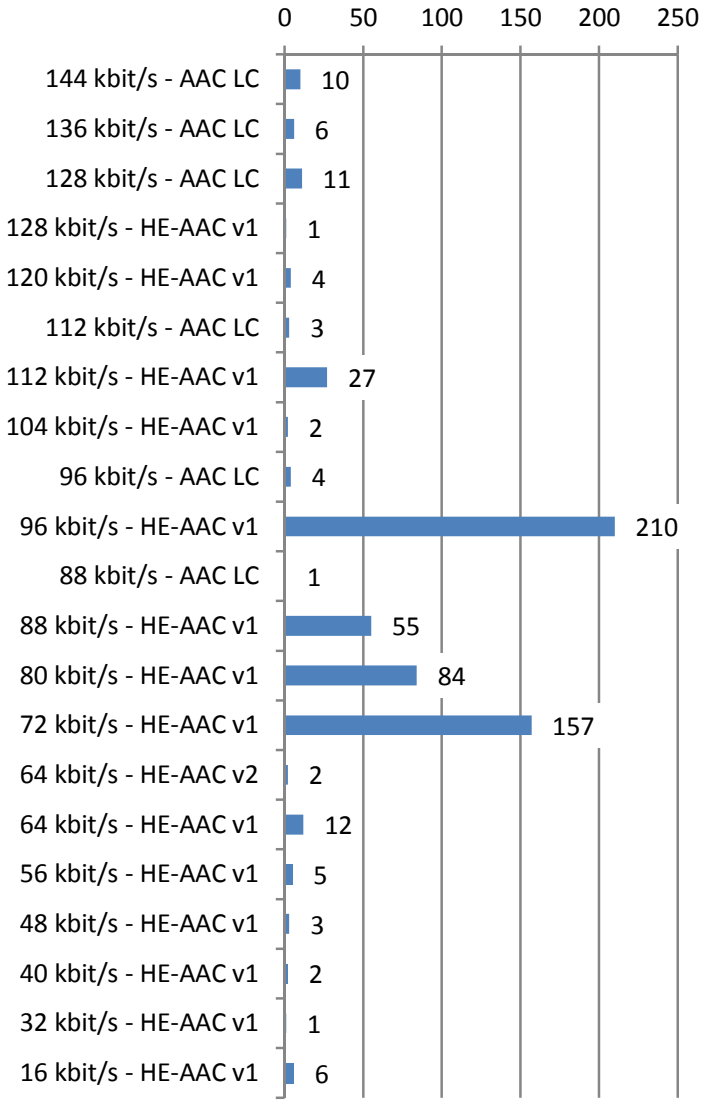

Fig. 10. Configurations used for $\mathrm{DAB}+$ audio coding in Germany and their respective frequency of use.

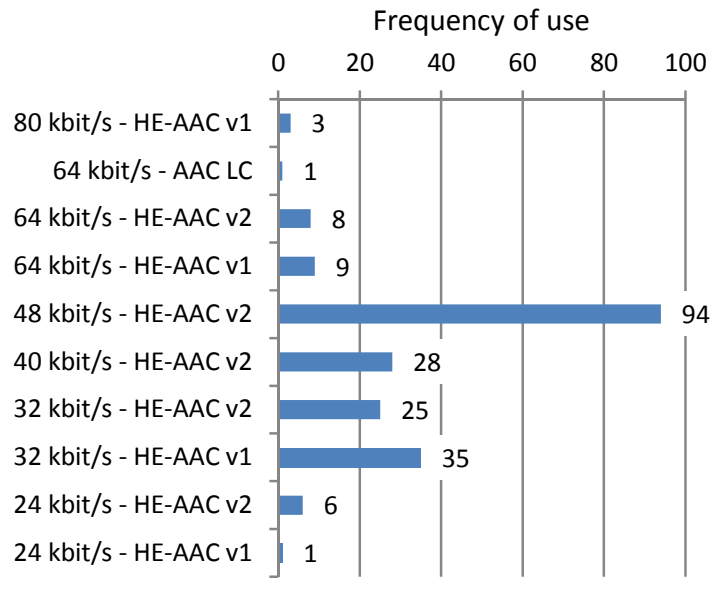

Fig. 11. Configurations used for $\mathrm{DAB}+$ audio coding in Great Britain and their respective frequency of use.

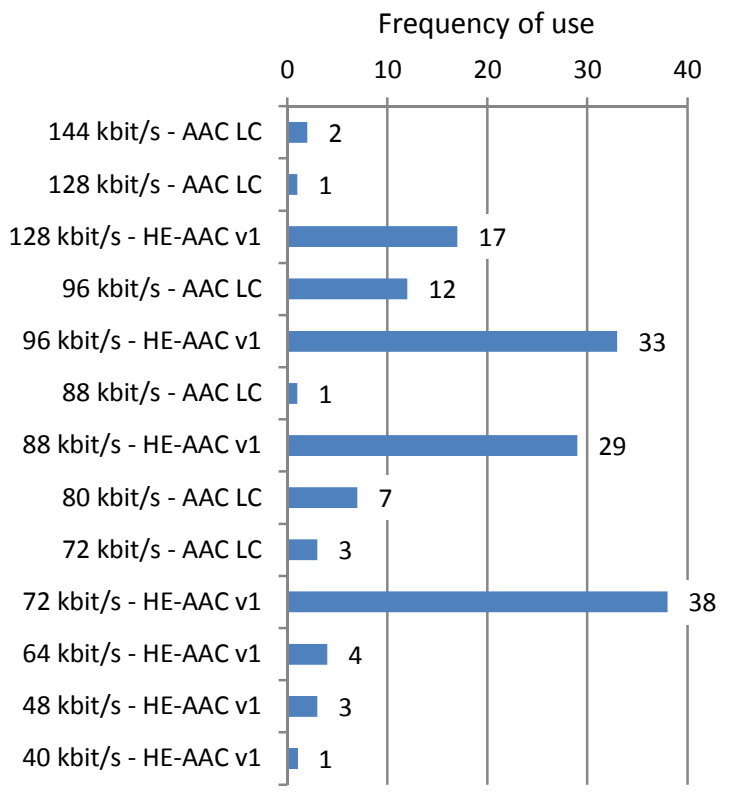

Fig. 12. Configurations used for $\mathrm{DAB}+$ audio coding in Denmark and their respective frequency of use.

The four dominant codecs can also be seen in Great Britain. They encode $87 \%$ of all $\mathrm{DAB}+$ radio stations. The most used codec is HE-AAC v2 with a bitrate of $48 \mathrm{kbps}$ (45\%), followed by the HE-AAC v1 codec in mono mode with $32 \mathrm{kbps}(17 \%)$ and two HE-AAC v2 codecs with the bitrates of $40 \mathrm{kbps}(13 \%)$ and $32 \mathrm{kbps}(12 \%)$ respectively. The remaining six configurations do not exceed $4 \%$ of each occurrence (see Fig. 11). In addition to $\mathrm{DAB}+$, a significant number of radio stations in Great Britain still use the original version of DAB with the MPEG 2 codec.

In Denmark, $\mathrm{DAB}+$ is dominated by five configurations, which, combined, cover $85 \%$ of all radio stations. Four of the most frequently used configurations utilize the HE-AAC v1 codec with a bitrate of $72 \mathrm{kbps}(25 \%)$, $96 \mathrm{kbps}(22 \%)$ and $88 \mathrm{kbps}(19 \%)$. A smaller representation has the same codec with a bitrate of $128 \mathrm{kbps}(11 \%)$ and the fifth most frequently used is the AAC LC codec with $96 \mathrm{kbps}(8 \%)$. The remaining eight configurations do not exceed $4 \%$ of each occurrence (see Fig. 12). 


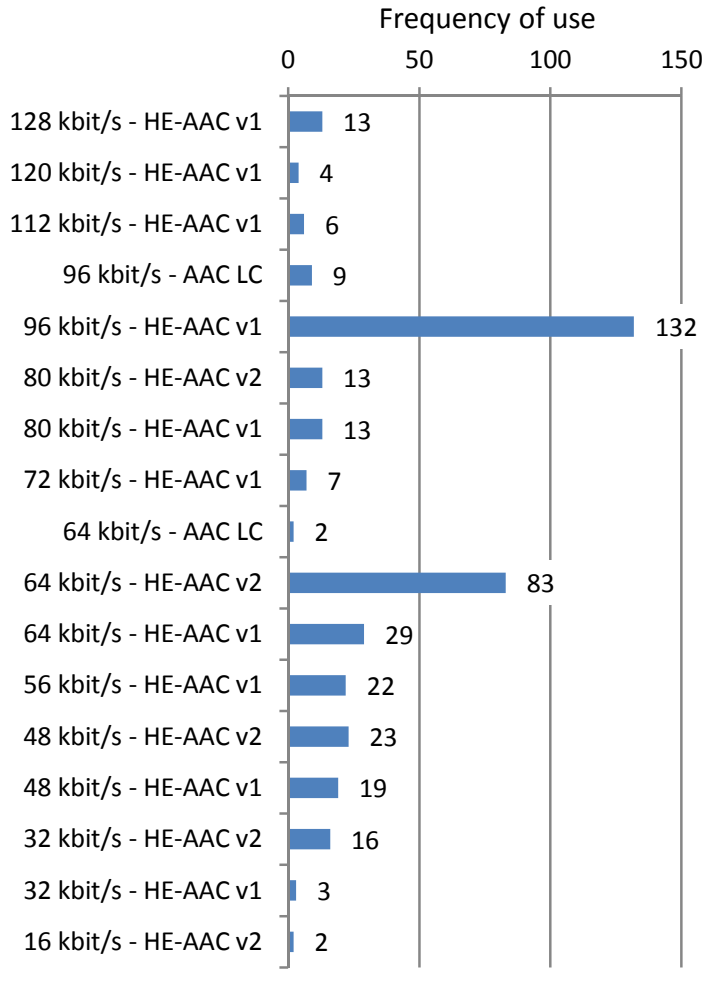

Fig. 13. Configurations used for $\mathrm{DAB}+$ audio coding in Italy and their respective frequency of use.

The situation in Italy is even more diverse. Although there is a clear preference for two configurations, a total of seven different audio codec configurations are used to cover $82 \%$ of radio stations. The HE-AAC v1 codec with a bitrate of $96 \mathrm{kbps}(33 \%)$ and HE-AAC v2 codec with a bitrate of $64 \mathrm{kbps}(21 \%)$ hold a significant dominance. The following configurations are then: HE-AAC v1 with $64 \mathrm{kbps}(7 \%)$, HE-AAC v2 with $48 \mathrm{kbps}(6 \%)$ and two HE-AAC v1 codecs with $56 \mathrm{kbps}(6 \%)$ and $48 \mathrm{kbps}(5 \%)$, as well as the HE-AAC v2 codec with $32 \mathrm{kbps}$ (4\%). Ten other codec configurations are used, but they do not exceed $3 \%$ of each occurrence (see Fig. 13).

\subsection{The Situation in the Czech Republic}

In the Czech Republic (the country where the author is implementing the $\mathrm{DAB}+$ system [13], [32]), the $\mathrm{DAB}+$ broadcasting is not yet fully established, mainly due to the absence of the relevant legislation. Although this platform is operated by four network operators, the only DAB+ multiplex that works with permanent, finalized parameters is the public service multiplex of Czech Radio. It covers 95\% of the population of the Czech Republic. This multiplex contains ten nationwide and ten regional radio stations. The HE-AAC v1 codec with a bitrate of $80 \mathrm{kbps}$ is used to encode the radio stations with a high share of music. Mainstream radio stations are coded using HE-AAC v2 codec with a bitrate of $64 \mathrm{kbps}$ and the radio stations with a high share of spoken word as well as regional stations are coded using HE-AAC v2 codec with 48 kbps (see Fig. 14).

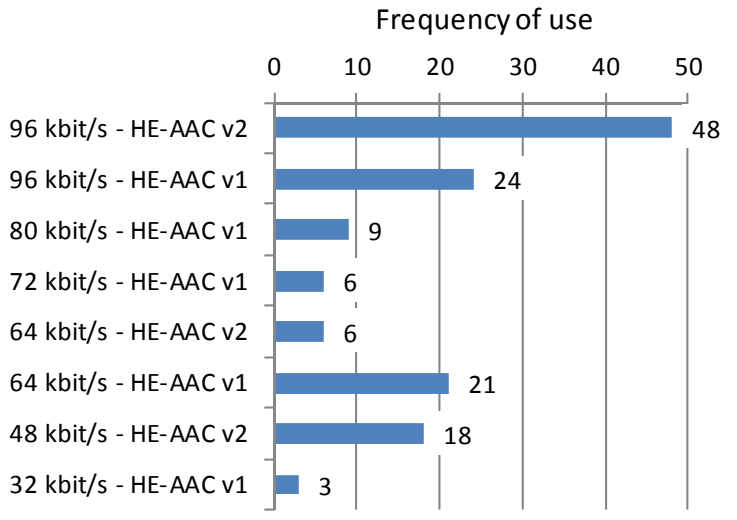

Fig. 14. Configurations used for $\mathrm{DAB}+$ audio coding in the Czech Republic and their respective frequency of use.

\section{Conclusion}

The sound in digital radio broadcasting is inevitably associated with lossy psychoacoustic compression. Compression algorithms are constantly evolving and being improved. Currently, the most efficient and state-of-the-art audio codec is HE-AAC v2, i.e. AAC with SBR and PS superstructures. It is able to deliver high quality sound even with a bitrate of $48 \mathrm{kbps}$, which is approximately four times less than the oldest codec of this group (MPEG 2) needs to achieve the same quality. However, the bitrate itself has almost no predictive value when it comes to the resulting sound quality. The aforementioned MUSHRA blind tests (see Sec. 3) confirmed that the decisive influence is made by the correct combination of the chosen compression algorithm and the corresponding bitrate. When the recommended bitrates are used for the relevant audio codecs, high subjectively perceived sound quality is achieved in all instances. This is also confirmed by the statistical results of the research on the behavior of European network operators, presented in Sec. 4. This research also clearly shows that the most used configuration of the $\mathrm{DAB}+$ codec in practice is HE-AAC v1 with a bitrate in the range of $64 \mathrm{kbps}-96 \mathrm{kbps}$, which covers more than $75 \%$ of DAB + broadcasting. Despite low bitrates, it is used for broadcasting of all genres. The results also show that the configurations of the codecs, across all European countries in the test, fall into the range of optimal bitrates (see Sec. 3), where they provide maximum efficiency and sound quality.

Because of the implementation of the $\mathrm{DAB}+$ platform, the broadcasting chain, i.e. the path from a recording studio to listeners, is becoming completely digitalized. The broadcasting chain can be divided into several stages, the basic ones being: the production of distribution media, processing in broadcasting companies, processing by the output broadcast audio processor and the signal transmission itself. In each of these stages, the lossy encoding of the audio is applied as well as its lossy decoding. In the digital broadcasting chain, such a process is then concate- 
nated several times, usually using various different algorithms. The examination of the DAB related audio codecs from the perspective of the entire broadcast system as a comprehensive specific environment has shown that the resulting subjectively perceived sound quality is affected by the following three key factors much more than by the bitrate level itself. The key factors are: 1) the technical quality of the input signal, 2) multiple cascading of the lossy non-entropic audio codecs within the entire digital broadcasting chain, and 3) the correct choice of codec configurations and their respective parameters.

\section{Acknowledgments}

The author would like to thank Zdeněk Otčenášek, Ph.D. (HAMU, MARC) and Pavel Straňák, Ph.D. (Czech Radio). This work was supported by the MŠMT ČR in the Long Term Conceptual Development of Research Institutes grant of the AMU in Prague: The 'Sound quality' project.

\section{References}

[1] HOEG, W., LAUTERBACH, T. (Eds.) Digital Audio Broadcasting: Principles and Applications of DAB, DAB+ and $D M B .3^{\text {rd }}$ ed. John Wiley \& Sons, 2009. ISBN:978-0-470-51037-7

[2] ETSI ETSI European Standard EN 300401 V2.1.1 Radio Broadcasting Systems; Digital Audio Broadcasting (DAB) to Mobile, Portable and Fixed Receivers. January 2017.

[3] O' NEILL, B. DAB Eureka-147: The European platform for digital radio. New Media Society, 2009, vol. 11, no. 1-2, p. 261-278. DOI: $10.1177 / 1461444808099578$

[4] BOWER, A., J. BBC digital radio - The Eureka 147 DAB system. Electronic Engineering, April 1998, p. 55-56. [Online] Available at: http://downloads.bbc.co.uk/rd/pubs/reports/1998-10.pdf

[5] GILSKI, P. DAB vs DAB+ radio broadcasting: A subjective comparative study. Archives of Acoustics, 2017, vol. 42, no. 4, p. 715-723. DOI: $10.1515 /$ aoa-2017-0074

[6] JAIN, P., SHARMA, S. Efficient performance analysis of OFDM based DAB systems using Reed Solomon coding technique. IOSR Journal of Electronics and Communication Engineering, 2015, vol. 10, no. 4, p. 56-59. DOI: 10.9790/2834-10435659

[7] ETSI ETSI Technical Specification TS 102 563, Digital Audio Broadcasting (DAB); Transport of Advanced Audio Coding (AAC) Audio. Sophia Antipolis Cedex, France, 2010.

[8] ETSI ETSI European Standard EN 302755 V1.4.1 Digital Video Broadcasting (DVB); Frame Structure Channel Coding and Modulation for a Second Generation Digital Terrestrial Television Broadcasting System (DVB-T2). July 2015.

[9] HERRE, J., DICK, S. Psychoacoustic models for perceptual audio coding - A tutorial review. Applied Sciences, 2019, vol. 9, no. 14, p. 1-22. DOI: $10.3390 /$ app 9142854

[10] MUIN, F. A., GUNAWAN, T. S., KARTIWI, M., et al. A review of lossless audio compression standards and algorithms. AIP Conference Proceedings, 2017, vol. 1883, no. 1, p. 1-12. DOI: $10.1063 / 1.5002024$
[11] HANS, M., SCHAFER, R. F. Lossless compression of digital audio. IEEE Signal Processing Magazine, 2001, vol. 18, no. 4, p. 21-32. DOI: $10.1109 / 79.939834$

[12] ITU RADIOCOMMUNICATION SECTOR, GENEVA SWITZERLAND. Method for the Subjective Assessment of Intermediate Quality Levels of Coding Systems. Recommendation ITU-R BS.1534. 2001-2015. Approved in 2015-10.

[13] ZYKA, K. DAB+ network implementation in the Czech Republic and impact of the audio coding on subjective perception of sound quality. Radioengineering, 2020 , vol. 29 , no. 1, p. 235-242. DOI: $10.13164 / \mathrm{re} .2020 .0235$

[14] ULOVEC, K., SMUTNY, M. Perceived audio quality analysis in digital audio broadcasting plus system based on PEAQ. Radioengineering, 2018, vol. 27 , no. 1, p. 342-352. DOI: $10.13164 / \mathrm{re} .2018 .0342$

[15] GILSKI, P., STEFAŃSKI, J. Subjective and objective comparative study of DAB+ broadcast system. Archives of Acoustics, 2017, vol. 42 , no. 1 , p. 3-11. DOI: 10.1515/aoa-2017-0001

[16] ETSI ETSI Technical Specification TS 103466 V1.1.1. Digital Audio Broadcasting (DAB); DAB Audio Coding (MPEG Layer II). $10 / 2016$.

[17] BRANDENBURG, K. MP3 and AAC explained. In AES $17^{\text {th }}$ International Conference on High Quality Audio Coding. Florence (Italy), 1999, p. 1-12.

[18] NOLL, P. MPEG Digital Audio Coding Standards. CRC Press LLC, 1999. [Online] Available at: https://doc.lagout.org/dsp/40.pdf

[19] INTERNATIONAL STANDARD, GENEVA SWITZERLAND. Information technology - Coding of Moving Pictures and Associated Audio for Digital Storage Media at up to about 1,5 Mbit/s - Part 3: Audio. Reference number ISO/IEC 111723:1993(E). August 1993.

[20] DEHERY, Y. F., LEVER, M., URCUN, P. A MUSICAM source codec for digital audio broadcasting and storage. In ICASSP 91: 1991 International Conference on Acoustics, Speech, and Signal Processing. Toronto (Canada), 1991, vol. 5, p. 3605-3608. DOI: 10.1109/ICASSP.1991.151054

[21] BRANDENBURG, K., POPP, H. An introduction to MPEG Layer-3. EBU Technical Review, 2000, p. 1-15. [Online] Available at: https://tech.ebu.ch/docs/techreview/trev_283-popp.pdf

[22] HERRE, J., DIETZ, M. MPEG-4 high-efficiency AAC coding [Standards in a Nutshell]. IEEE Signal Processing Magazine, 2008, vol. 25, no. 3, p. 137-142. DOI: 10.1109/MSP.2008.918684

[23] ITU RADIOCOMMUNICATION SECTOR, GENEVA SWITZERLAND. Audio Coding for Digital Broadcasting. BS Series Broadcasting Service (Sound). Recommendation ITU-R BS.1196-8. 2019. Approved in 2019-10.

[24] INTERNATIONAL STANDARD, GENEVA SWITZERLAND. Information Technology - Generic Coding of Moving Pictures and Associated Audio Information - Part 7: Advanced Audio Coding $(A A C)$. Reference number ISO/IEC 13818-7:2004(E), 3rd ed. October 2004

[25] INTERNATIONAL STANDARD, GENEVA SWITZERLAND. Information Technology - Coding of Audio-Visual Objects Part 3: Audio. Reference number ISO/IEC 14496-3(E). $2^{\text {nd }}$ ed, December 2001.

[26] WANG, Y., VILERMO, M. Modified discrete cosine transform Its implications for audio coding and error concealment. In AES 22nd International Conference on Virtual, Synthetic and Entertainment Audio. Finland, 2002, paper no. 258, p. 1-10.

[27] HERRE, J., JOHNSTON, J. D. Enhancing the performance of perceptual audio coders by using Temporal Noise Shaping (TNS). 
Presented at AES 101st Convention. Los Angeles (USA), 1996, paper no. 4384, p. 1-24.

[28] DIETZ, M., LILJERYD, L., KJÖRLING, K., et al. Spectral Band Replication, a novel approach in audio coding. Presented at $A E S$ 112 th Convention. Munich (Germany), May 2002, paper no. 5553, p. 1-8.

[29] ITU RADIOCOMMUNICATION SECTOR, GENEVA SWITZERLAND. Audio Coding for Digital Broadcasting. Recommendation ITU-R BS. 1196-7. 1995-2019. Approved in 2019-10.

[30] MELTZER, S., MOSER, G. MPEG-4 HE-AAC v2 - audio coding for today's media world. EBU Technical Review, 2006. [Online] Available at: https://tech.ebu.ch/docs/techreview/trev_305moser.pdf

[31] BREEBAART, J., VAN DE PAR, S., KOHLRAUSCH, A., et al. Parametric Coding of Stereo Audio. EURASIP Journal on Applied Signal Processing, 2005, p. 1305-1322. DOI: 10.1155/ASP.2005.1305

[32] ZYKA, K. The digital audio broadcasting journey from the lab to listeners - the Czech Republic case study. Radioengineering, 2019, vol. 28, no. 2, p. 483-490. DOI: 10.13164/re.2019.0483

[33] SONTACCHI, A., POMBERGER, H., HÖLDRICH, R. Recruiting and evaluation process of an expert listening panel. In the International Conference on Acoustics NAG/DAGA. Rotterdam (the Netherlands), 2009, p. 1552-1555. [Online] Available at: http: //pub.dega-akustik.de/NAG_DAGA_2009/data/articles/000494.pdf

[34] STRANAK, P. Interfering DC component, suppression and influence to digital signal processing. Radioengineering, 2008, vol. 17, no. 3, p. 121-123. ISSN: 1210-2512

[35] STRANAK, P., DOBES, J. Controlling peaks of the audio signal by dynamically allocated scale factor for lossy psychoacoustic encoder. In 53rd IEEE International Midwest Symposium on
Circuits and Systems. Seattle (USA), 2010, p. 388-391. DOI: 10.1109/MWSCAS.2010.5548874

[36] FMLIST - The Database of Worldwide FM Radio, DAB and TV. [Online] Available at: https://www.fmlist.org/ul_login.php

[37] FMLIST - DAB Ensembles Worldwide. [Online] Available at: http://www.fmlist.org/sendertabelle/dab-ww.php

[38] WORLD DAB - The Official Database of the Latest Information on Regulatory Frameworks, DAB+ Network Coverage, Services on Air. [Online] Available at: https://www.worlddab.org/countries

[39] EBU - Digital Radio Report 2020. [Online] Available at: https://www.ebu.ch/publications/research/login_only/report/digital -radio

\section{About the Author ...}

Karel ZÝKA was born in Prague, the Czech Republic, in 1967. He received his M.Sc. from the Faculty of Electrical Engineering at the Czech Technical University in Prague in 1992. He is employed at Czech Radio, a national public service broadcaster, where he is the Technical Director and the head of the digital switchover team, which is implementing the $\mathrm{DAB}+$ in the Czech Republic. He is a member of WorldDAB and EDRA Steering Boards and works for the EBU. In the past, he was engaged at Radio Alfa, implementing the first satellite distribution system in the Czech Republic, and at TV Prima, where he participated in the DVB-T transformation. Currently, he is an external postgraduate $\mathrm{PhD}$ student at the Academy of Performing Arts in Prague (HAMU), Sound Design Department. 